\title{
Eficiência da seleção para incremento do teor de óleo do pinhão-manso
}

\author{
Rodrigo Barros Rocha(1), André Rostand Ramalho(1), Alexsandro Lara Teixeira(1), Bruno Galvêas Laviola(2), \\ Francisco Célio Gomes da Silva ${ }^{(3)}$ e Júlio Sancho Linhares Teixeira Militão(3)
}

(1)Embrapa Rondônia, BR 364, Km 5,5, Zona Rural, CEP 76815-800 Porto Velho, RO. E-mail: rodrigo@cpafro.embrapa.br, rostand@cpafro.embrapa.br, alexteixeira@cpafro.embrapa.br ${ }^{(2)}$ Embrapa Agroenergia, Parque Estação Biológica, Avenida W3 Norte Final, Asa Norte, CEP 70770-901 Brasília, DF. E-mail: bruno.laviola@embrapa.br ${ }^{(3)}$ Universidade Federal de Rondônia, Campus BR 364, Km 9,5, Zona Rural,CEP 78900-000 Porto Velho, RO. E-mail: celiohermoso@gmail.com, militao@unir.br

\begin{abstract}
Resumo - O objetivo deste trabalho foi estimar os parâmetros genéticos de características de qualidade de grãos de pinhão-manso (Jatropha curcas) e quantificar o progresso genético da seleção. O teor de óleo dos grãos e os pesos de grãos, amêndoas e casca de 120 plantas, selecionadas ao acaso em plantio comercial, foram avaliados aos 36 e 48 meses de cultivo. O progresso genético foi quantificado por meio da avaliação dos ganhos diretos e da utilização de índices de seleção. O efeito da interação genótipos x medição foi significativo para todas as características avaliadas; portanto, há genótipos com comportamento diferenciado nas duas colheitas. Os parâmetros genéticos indicaram predominância da variação genotípica na expressão das características avaliadas, e observou-se tendência de manutenção da superioridade dos genótipos ao longo do tempo. O índice de seleção genótipo-ideótipo resultou em maior ganho total e promoveu alteração mais equilibrada na média das características. A seleção direta para peso de amêndoa resulta em ganhos totais semelhantes aos obtidos com a utilização dos índices de seleção.
\end{abstract}

Termos para indexação: Jatropha curcas, biocombustível, índice de seleção, melhoramento genético, peso de amêndoas, variedade comercial.

\section{Selection efficiency for increasing physic nut oil content}

\begin{abstract}
The objective of this work was to estimate the genetic parameters of physic nut (Jatropha curcas) grain quality traits and to quantify the genetic progress with selection. Seed oil content, and seed, kernel, and shell weights of 120 plants, randomly selected in a commercial planting, were evaluated at 36 and 48 months. Genetic progress was quantified by the evaluation of direct gains and by the use of selection indexes. The genotype $\mathrm{x}$ measurement interaction was significant for all evaluated traits; therefore, there are genotypes with different behavior in the two cropping seasons. The genetic parameters indicated a higher proportion of genotypic variation in the expression of the evaluated traits, and a trend of the genotypes to maintain their genetic superiority over time was observed. The genotype-ideotype selection index resulted in higher genetic gains and promoted a more balanced alteration in the trait means. The direct selection for kernel weight results in genetic gains similar to those obtained with the use of the selection indexes.
\end{abstract}

Index terms: Jatropha curcas, biofuels, selection index, genetic breeding, kernel weight, commercial variety.

\section{Introdução}

Entre as espécies oleaginosas com potencial para produção de biodiesel, destaca-se o pinhão-manso (Jatropha curcas L.), uma espécie perene, diploide, monoica e pertencente à família Euforbiaceae (Acthen et al., 2010). Essa oleaginosa vem sendo pesquisada como fonte potencial de matéria-prima para a produção de biocombustíveis (Oliveira \& Ramalho, 2006).

A espécie apresenta algumas características desejáveis, tais como óleo de alta qualidade e possibilidade de inserção na cadeia produtiva da agricultura familiar (Laviola et al., 2010). Contudo, limitações técnicas têm impedido a inserção plena do pinhão-manso na matriz energética brasileira. A maturação desuniforme dos frutos e a baixa produtividade de grãos têm limitado a viabilidade econômica desse cultivo. Da expectativa inicial de quatro ou mais toneladas de grãos por hectare, produtividades inferiores a 2,0 $\mathrm{Mg} \mathrm{ha}^{-1}$ estão sendo obtidas em diferentes condições edafoclimáticas, principalmente em razão de limitações hídricas ou do ataque de pragas e doenças. Projeções não realizadas da produtividade de grãos, podem ser atribuídas à observações de plantas isoladas, que desconsiderem a redução no desenvolvimento em plantios. Spinelli et al. (2010), ao quantificar os efeitos diretos e indiretos de características vegetativas e da qualidade da matéria-prima no rendimento de óleo do

Pesq. agropec. bras., Brasília, v.47, n.1, p.44-50, jan. 2012 
pinhão-manso, observaram que a produtividade de grãos foi o componente mais importante do rendimento de óleo, seguida do volume de copa e do teor de óleo nos grãos.

O melhoramento do pinhão-manso, ainda considerado como uma planta em domesticação, está em seus estágios iniciais. O conhecimento do controle genético da herança dos componentes de produção é fundamental para o estabelecimento de estratégias eficientes de seleção e para o desenvolvimento de variedades comerciais. Para isso, é essencial a análise da herdabilidade, das correlações genéticas e da repetibilidade (Resende, 2002).

A magnitude e o sentido das correlações genéticas fazem com que a seleção baseada em uma única característica resulte em alterações não desejadas em outros componentes de produção. Essas alterações são chamadas de respostas correlacionadas e o seu sentido deve ser considerado na seleção de plantas (Cruz et al., 2004). Kaushik et al. (2007) observaram a ocorrência de correlação positiva e de alta magnitude entre peso de sementes e teor de óleo. Em outros estudos, o teor de óleo foi determinado a partir de amostragens aleatórias de várias regiões, tendo-se realizado inferências sobre o efeito ambiental nesta característica (Ginwal et al., 2005). Entretanto, são escassos os trabalhos que quantifiquem os componentes genéticos e o ganho com a seleção levando-se em consideração medidas repetidas e a avaliação de plantas em idade produtiva.

As características de qualidade da matéria-prima de $J$. curcas diferenciam-se principalmente quanto aos pesos de grãos, de amêndoas e de casca e ao teor de óleo nos grãos (Rao et al., 2008). As sementes dessa espécie apresentam tegumento rijo e quebradiço, que reveste a amêndoa, de albúmen branco, tenro e rico em óleo (Nunes et al., 2009). Segundo Heller (1996), as sementes, quando secas, apresentam de 1,5 a 2,0 cm de comprimento e de 1,0 a $1,5 \mathrm{~cm}$ de largura, bem como peso individual entre 0,5 e $0,8 \mathrm{~g}$, a depender da variedade e dos tratos culturais. De acordo com este autor, a semente apresenta, em média, proporções de $45 \%$ de casca e $55 \%$ de amêndoa. Arruda et al. (2004) relataram uma faixa de variabilidade para o teor de óleo de 25 a 40\%. Mais recentemente, ao utilizar métodos indiretos de análise, Freitas et al. (2011) quantificaram teores de óleo entre 16,0 e 45,6\%.

O objetivo deste trabalho foi estimar os parâmetros genéticos de características de qualidade de grãos de pinhão-manso e quantificar o progresso genético da seleção.

\section{Material e Métodos}

Foram caracterizadas amostras de frutos de 120 plantas selecionadas ao acaso dentro de uma população de pinhão-manso em plantio comercial, aos 36 e 48 meses de cultivo, no Município de Ariquemes, RO (9 $55^{\prime} 24^{\prime \prime S}$ e $63^{\circ} 7^{\prime} 15^{\prime \prime} \mathrm{W}$, a $142 \mathrm{~m}$ de altitude). O clima da região, conforme a classificação de Köppen, é do tipo Aw, tropical, quente e úmido, com período seco bem definido e ocorrência de deficit hídrico no período de junho a setembro. As variáveis climáticas são: temperatura média anual de $25^{\circ} \mathrm{C}$, precipitação média de $2.200 \mathrm{~mm}$ e evapotranspiração média de $851 \mathrm{~mm}$, de acordo com as Normais Climatológicas (1992). O solo da área experimental é classificado como Latossolo Vermelho-Amarelo distrófico, de textura muito argilosa.

O plantio foi realizado em espaçamento $3 \times 2 \mathrm{~m}$, em covas de 20 a $30 \mathrm{~cm}$ de profundidade, com mudas de 30 dias de crescimento em viveiro, acondicionadas em sacolas de plástico de $2 \mathrm{~L}$. A origem genética das plantas matrizes é desconhecida. A adubação química inicial consistiu de $100 \mathrm{~g}$ de superfosfato simples por cova. Aos 12 meses pós-plantio, foi realizada calagem superficial em área total, com a aplicação de $4 \mathrm{Mg} \mathrm{ha}^{-1}$ de calcário (PRNT 60\%). A partir do segundo ano, iniciou-se adubação química de cobertura com a aplicação de $50 \mathrm{~g}$ de $\mathrm{N}, 60 \mathrm{~g}$ de $\mathrm{P}_{2} \mathrm{O}_{5}$ e $40 \mathrm{~g}$ de $\mathrm{K}_{2} \mathrm{O}$ por planta, duas vezes ao ano (abril e outubro). No decorrer do experimento, amostras de solo foram coletadas e encaminhadas ao Laboratório de Análises de Solos e Plantas da Embrapa Rondônia (Tabela 1).

As características de qualidade dos grãos foram avaliadas no Laboratório de Extração de Óleos da Universidade Federal de Rondônia, Porto Velho, RO. Os procedimentos para extração de óleo foram realizados com o trituramento das sementes inteiras e dos albúmens, de forma separada, com a quantificação gravimétrica dos pesos de grãos, de amêndoa e de casca (epicarpo e mesocarpo). As análises foram realizadas de acordo com as normas do Instituto Adolfo Lutz (1985), que compreendem umidade e cinzas. As bateladas de extrações do óleo foram realizadas com uso de duas réplicas por amostra, em extrator de Soxhlet, tendo-se utilizado hexano como solvente, com tempo de 4 horas 
de extração e $200 \mathrm{~g}$ de amostra. Após a extração do óleo, foram identificadas amostras que apresentaram diferença entre repetições igual ou superior a $1 \%$ do teor de óleo, as quais tiveram seu teor quantificado novamente.

Os valores genéticos das características peso de grãos, peso de amêndoa, peso de casca e teor de óleo foram estimados por meio do procedimento REML/Blup (Resende, 2002). A predição dos valores genéticos a partir do sistema de equações de modelos mistos presume o conhecimento dos componentes de variância que são estimados com uso do método de máxima verossimilhança restrita (REML).

A predição dos valores genotípicos foi feita separadamente, aos 36 e 48 meses de plantio, com uso do modelo linear misto, $\mathrm{y}=\mathrm{Xu}+\mathrm{Zg}+\varepsilon$ (Resende, 2002) em que: y é o vetor de dados; u é o valor escalar referente à média geral (efeito fixo); g é o vetor dos efeitos genotípicos, assumidos como aleatórios; e $\varepsilon$ é o vetor de erros aleatórios ou resíduos. As letras maiúsculas representam as matrizes de incidência para esses efeitos.

A predição dos parâmetros genéticos, ao se considerar o efeito permanente do ambiente e a correlação fenotípica entre medidas repetidas de um mesmo indivíduo, foi realizada com o modelo (Resende, 2002) $\mathrm{y}=\mathrm{Xm}+\mathrm{Wp}+\varepsilon$, em que: $\mathrm{y}$, vetor de dados; $\mathrm{m}$, vetor dos efeitos fixos de medição somados à média geral; $p$, vetor dos efeitos aleatórios de genótipos somados aos efeitos permanentes de ambiente; e $\varepsilon$, vetor de erros ou resíduos, assumidos como aleatórios. As letras maiúsculas representam as matrizes de incidência para esses efeitos. A significância dos efeitos de genótipo, de medições e da interação genótipos $\mathrm{x}$ medições foi avaliada pelo teste $\mathrm{F}$, a $1 \%$ de probabilidade.
A repetibilidade individual $\left(\hat{\rho}_{\mathrm{i}}\right)$ foi estimada a partir das correlações fenotípicas entre medições repetidas em um mesmo indivíduo, por meio da expressão $\hat{\rho}_{\mathrm{i}}=\hat{\sigma}_{\mathrm{gp}}^{2} /\left(\hat{\sigma}_{\mathrm{gp}}^{2}+\hat{\sigma}^{2}\right)$, em que: $\hat{\sigma}_{\mathrm{gp}}^{2}$ é a variância genotípica adicionada aos efeitos permanentes de ambiente, e $\hat{\sigma}^{2}$ é a variância residual. A repetibilidade média $\left(\rho_{\text {im }}\right)$ da população foi calculada com base na expressão: $\rho_{\mathrm{im}}=m \rho_{\mathrm{i}} /\left[1+(\mathrm{m}-1) \rho_{\mathrm{i}}\right]$, em que $\mathrm{m}$ é o número de avaliações.

A acurácia de seleção $\left(\mathrm{r}_{\mathrm{p} p}\right)$, definida como a correlação entre o valor genético verdadeiro do indivíduo e o valor fenotípico, foi estimada por meio da expressão: $\rho_{\hat{p p}}=\left\{\mathrm{m} \rho_{\mathrm{i}} /\left[1+(\mathrm{m}-1) \rho_{\mathrm{i}}\right]\right\}^{0,5}$. A eficiência do uso de medidas repetidas foi determinada a partir da expressão: $\mathrm{E}=\left\{\mathrm{m} /\left[1+(\mathrm{m}-1) \rho_{1}\right]\right\}^{0,5}$.

$\mathrm{O}$ progresso genético foi quantificado, tendo-se considerado os ganhos diretos, a resposta correlacionada e a utilização de índices de seleção. As estimativas do ganho direto $\left(\mathrm{G}_{\mathrm{s}}\right)$, que mensura o progresso genético com a seleção na própria característica, foram obtidas com a expressão (Cruz et al., 2004): $\mathrm{Gs}=\mathrm{k} \times \mathrm{r}_{\mathrm{pp}} \times \sigma_{\mathrm{gp}}$, em que $\mathrm{k}$ é o diferencial de seleção padronizado.

A resposta correlacionada (RC), que mensura a alteração em caracteres relacionados à seleção em uma característica principal, foi estimada levando-se em consideração avaliações nas duas colheitas, conforme a expressão: $\mathrm{RC}(\mathrm{y} / \mathrm{x})=\mathrm{k} \times \mathrm{r}_{\mathrm{g}(\mathrm{x}, \mathrm{y})} \times \rho_{\mathrm{ix}} \times \rho_{\mathrm{iy}} \times \sigma_{\mathrm{gpy}}$.

Os valores genotípicos foram utilizados para quantificar o progresso genético por meio do uso do índice livre de peso e parâmetros (Elston, 1963), do índice baseado na soma de postos (Mulamba \& Mock, 1978) e do índice genótipo-ideótipo (Cruz, 2004).

A classificação dos genótipos no índice livre de peso e parâmetros (Elston, 1963) é dada por uma combinação entre os valores genotípicos e os máximos

Tabela 1. Variação nos resultados da análise química do solo ao longo do período experimental ${ }^{(1)}$.

\begin{tabular}{|c|c|c|c|c|c|c|c|c|c|}
\hline $\begin{array}{l}\text { Profundidade } \\
(\mathrm{cm})\end{array}$ & $\begin{array}{c}\mathrm{pH} \\
\text { em água }\end{array}$ & $\begin{array}{c}\mathrm{P} \\
\left(\mathrm{mg} \mathrm{dm}^{-3}\right) \\
\end{array}$ & K & $\mathrm{Ca}$ & $\begin{array}{c}\mathrm{Mg} \\
\mathrm{mol}_{\mathrm{c}} \mathrm{d}\end{array}$ & $\mathrm{H}+\mathrm{Al}$ & $\begin{array}{c}\mathrm{Al} \\
----- \\
\end{array}$ & $\begin{array}{c}\mathrm{MO} \\
\left(\mathrm{g} \mathrm{kg}^{-1}\right)\end{array}$ & $\begin{array}{c}\mathrm{V} \\
(\%) \\
\end{array}$ \\
\hline & \multicolumn{9}{|c|}{ Setembro de 2008} \\
\hline $0-20$ & 4,2 & 0,4 & 1,1 & 7,0 & 6,0 & 41,0 & 20,0 & 13,0 & 21,0 \\
\hline \multirow[t]{2}{*}{$20-40$} & 4,5 & 2,0 & 0,3 & 5,1 & 4,0 & 61,0 & 10,1 & 9,2 & 13,0 \\
\hline & \multicolumn{9}{|c|}{ Setembro de 2009} \\
\hline $0-20$ & 5,2 & 16,0 & 1,2 & 27,0 & 17,0 & 57,8 & 0,0 & 19,6 & 44,0 \\
\hline \multirow[t]{2}{*}{$20-40$} & 4,2 & 1,0 & 0,4 & 5,0 & 4,1 & 57,8 & 9,1 & 9,5 & 13,0 \\
\hline & \multicolumn{9}{|c|}{ Setembro de 2010} \\
\hline $0-20$ & 5,4 & 9,0 & 1,0 & 33,3 & 15,0 & 52,8 & 0,0 & 17,6 & 49,0 \\
\hline $20-40$ & 4,6 & 1,0 & 0,3 & 5,0 & 4,5 & 62,7 & 10,7 & 11,1 & 14,0 \\
\hline
\end{tabular}

${ }^{(1)} \mathrm{MO}$, matéria orgânica do solo; V, saturação por bases. 
e mínimos observados, $\mathrm{I}_{\mathrm{e}}=\mathrm{W}_{1} \times \mathrm{W}_{2} \times \mathrm{W}_{3} \ldots \times \mathrm{W}_{\mathrm{n}}$, em que: $\mathrm{w}_{\mathrm{j}}=\mathrm{x}_{\mathrm{ij}}-\mathrm{k}_{\mathrm{j}}, \mathrm{x}_{\mathrm{ij}}$ é o valor do i-ésimo genótipo no j-ésimo caráter, e $\mathrm{k}_{\mathrm{j}}$ é o limite mínimo ou máximo previamente estabelecido para cada característica.

O índice baseado na soma de postos (Mulamba \& Mock, 1978) corresponde ao somatório da classificação (postos) dos genótipos, ordenados de acordo com o seu valor genético para cada uma das características. A classificação dos genótipos foi obtida com o ordenamento em ordem decrescente dos valores genéticos das características avaliadas.

No índice genótipo-ideótipo (Cruz et al., 2004), são consideradas as distâncias estimadas entre os genótipos e os valores referências, definidos pelos máximos e mínimos observados, conforme a expressão:

$$
\mathrm{G}_{\mathrm{i}}=\left[1 / \mathrm{n} \sum_{\mathrm{j}=1}^{\mathrm{n}}\left(\mathrm{x}_{\mathrm{ij}}-\mathrm{m}_{\mathrm{j}}\right)^{2}\right]^{0,5}
$$

em que: $\mathrm{G}_{\mathrm{i}}$ é a distância genótipo-ideótipo; $\mathrm{x}_{\mathrm{ij}}$ é o escore da técnica de componentes principais do i-ésimo genótipo no j-ésimo componente principal; e $\mathrm{m}_{\mathrm{j}}$ é o escore associado à referência ideal no j-ésimo componente principal.

\section{Resultados e Discussão}

$\mathrm{O}$ efeito da interação genótipos $\mathrm{x}$ medição foi significativo para todas as características avaliadas, o que indica a existência de genótipos com desempenho diferenciado aos 36 e 48 meses de plantio (Tabela 2). O desempenho diferenciado dos genótipos ao longo dos anos é uma característica típica de espécies perenes e é causado pela influência do ambiente na expressão dos caracteres.

Tabela 2. Resumo da análise de variância das características de qualidade de grãos de Jatropha curcas.

\begin{tabular}{lccccc}
\hline Fonte de & GL & \multicolumn{3}{c}{ Peso } & Teor de \\
\cline { 3 - 5 } variação & & Casca & Amêndoa & Grãos & óleo \\
\hline Genótipos (G) & 119 & $8,859^{* *}$ & $13,560^{* *}$ & $14,330^{* *}$ & $11,37^{* *}$ \\
Medição (M) & 1 & $0,481^{\text {ns }}$ & $4,391^{* *}$ & $1,838^{\text {ns }}$ & $76,49^{* *}$ \\
G x M & 119 & $3,035^{* *}$ & $5,378^{* *}$ & $5,410^{* *}$ & $6,38^{* *}$ \\
Resíduo & 240 & - & - & - & - \\
Total & 479 & - & - & - & - \\
\hline Média & - & 4,3498 & 7,2186 & 11,5686 & 32,62 \\
\hline
\end{tabular}

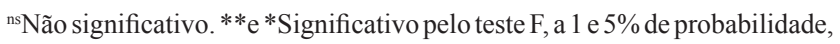
respectivamente.
Especificamente, o efeito das medições foi significativo para peso da amêndoa e teor de óleo (Tabela 2). Essas características dependem da taxa de acúmulo de matéria seca durante o estádio de enchimento de grãos, que é determinado pela expressão dos genes influenciada pelo ambiente. Melhorias nas condições de cultivo, proporcionadas pela calagem em área total e pela adubação química em cobertura, foram observadas aos 36 e 48 meses de plantio (Tabela 1).

$\mathrm{O}$ teor de óleo nos grãos apresentou variabilidade genética significativa pelo teste $\mathrm{F}$ (Tabela 2). Resultados semelhantes foram obtidos em outras populações de pinhão-manso, como observado por Kaushik et al. (2007), que verificaram diferenças significativas no teor de óleo de 24 acessos provenientes da Índia. A ocorrência de variabilidade genética significativa entre as plantas é fundamental para o progresso genético por meio da seleção e depende da contribuição significativa do efeito do genótipo para a expressão da característica.

A média geral do teor de óleo observada no plantio (32\%) é comparável à média de $34,5 \%$, quantificada por Heller (1996), e à de 31\%, obtida por Freitas et al. (2011). Foi observada amplitude de variação de 23,3 a $39,0 \%$, aos 36 meses, e de 27,1 a 40,2\%, aos 48 meses. Kaushik et al. (2007) relataram faixa de variabilidade entre 28,8 e $38,9 \%$. A média e a dispersão dos valores indicam representatividade dos materiais avaliados em relação à variabilidade descrita para a espécie na literatura. Recentemente, novos acessos têm sido caracterizados na América Central, centro de origem dessa oleaginosa. Quintero et al. (2011) descreveram genótipos provenientes da América Central que apresentam teor de óleo superior a $50 \%$.

As estimativas do efeito aleatório de genótipo e do efeito permanente do ambiente permitem quantificar a proporção de variação total atribuída à diferença entre os genótipos (Rocha et al., 2005). O coeficiente de variação genético, que mensura a fração da variância total em virtude da variação genotípica entre plantas, indicou predominância do componente genético, na expressão das características de qualidade (Tabela 3). De acordo com Resende (2002), os valores de herdabilidade $\left(\mathrm{h}^{2}\right)$ observados podem ser considerados de alta magnitude $\left(h^{2}>60 \%\right)$ e acurados, uma vez que os valores de desvio-padrão observados foram inferiores a $20 \%$ do valor das estimativas. A repetibilidade, que representa o limite superior da herdabilidade individual, 
indica a possibilidade de selecionar genótipos de maior adaptabilidade e estabilidade, ou seja, responsivos à melhoria do ambiente, e que mantêm sua superioridade ao longo dos anos (Tabela 4).

A acurácia de seleção, definida pela correlação entre o valor genético verdadeiro do indivíduo e o índice fenotípico utilizado para obtenção das estimativas dos componentes genéticos, foi interpretada para avaliar o número mínimo de avaliações necessárias à seleção de plantas. Segundo Resende (2002), valores de acurácia seletiva acima de $80 \%$ são adequados para a obtenção

Tabela 3. Parâmetros genéticos estimados das características de qualidade de grãos de Jatropha curcas, avaliadas aos 36 e 48 meses de plantio.

\begin{tabular}{|c|c|c|c|c|}
\hline \multirow{2}{*}{$\begin{array}{l}\text { Parâmetros } \\
\text { genéticos }^{(1)}\end{array}$} & \multicolumn{3}{|c|}{ Peso (g) } & \multirow{2}{*}{$\begin{array}{c}\text { Teor de } \\
\text { óleo }(\%)\end{array}$} \\
\hline & Casca & Amêndoa & Grãos & \\
\hline & \multicolumn{4}{|c|}{ Avaliação aos 36 meses } \\
\hline$\hat{\sigma}_{g}^{2}$ & 0,0690 & 0,3571 & 0,7206 & 0,00033 \\
\hline$\hat{\sigma}^{2}$ & 0,0259 & 0,1649 & 0,2604 & 0,00008 \\
\hline$\hat{\sigma}_{\mathrm{f}}^{2}$ & 0,0948 & 0,5221 & 0,9810 & 0,000412 \\
\hline $\mathrm{h}_{\mathrm{g}}^{2}$ & $0,727 \pm 0,1570$ & $0,684 \pm 0,1523$ & $0,734 \pm 0,1578$ & $0,795 \pm 0,1642$ \\
\hline $\mathrm{CV}_{\mathrm{g}}$ & 6,0238 & 8,3687 & 7,3810 & 5,7265 \\
\hline$\underline{\mathrm{CV}_{\mathrm{e}}}$ & 3,6898 & 5,6879 & 4,4373 & 2,9048 \\
\hline \multirow[t]{2}{*}{ Média } & 4,3598 & 7,1406 & 11,5005 & 31,6 \\
\hline & \multicolumn{4}{|c|}{ Avaliação aos 48 meses } \\
\hline$\hat{\sigma}_{\mathrm{g}}^{2}$ & 0,0904 & 0,6797 & 1,2398 & 0,0005 \\
\hline$\hat{\sigma}^{2}$ & 0,0385 & 0,0798 & 0,1816 & 0,0001 \\
\hline$\hat{\sigma}_{\mathrm{f}}^{2}$ & 0,1290 & 0,7596 & 1,4214 & 0,0006 \\
\hline $\mathrm{h}_{\mathrm{g}}^{2}$ & $0,700 \pm 0,1441$ & $0,894 \pm 0,1742$ & $0,872 \pm 0,1720$ & $0,787 \pm 0,1634$ \\
\hline $\mathrm{CV}_{\mathrm{g}}$ & 6,9296 & 11,2981 & 9,5686 & 6,3617 \\
\hline$\underline{\mathrm{CV}_{\mathrm{e}}}$ & 4,5262 & 3,8722 & 3,6616 & 3,3056 \\
\hline Média & 4,3397 & 7,2972 & 11,6368 & 33,7 \\
\hline
\end{tabular}

${ }^{(1)} \hat{\sigma}_{\mathrm{g}}^{2}$, variância genotípica; $\hat{\sigma}^{2}$, variância residual; $\hat{\sigma}_{\mathrm{f}}^{2}$, variância fenotípica; $\mathrm{h}_{\mathrm{g}}^{2}$, herdabilidade individual no sentido amplo; $\mathrm{CV}_{\mathrm{g}}$, coeficiente de variação genotípico; $\mathrm{CV}_{\mathrm{e}}$, coeficiente de variação ambiental.

Tabela 4. Estimativas de repetibilidade e acurácia seletiva de características de qualidade de grãos de Jatropha curcas.

\begin{tabular}{|c|c|c|c|c|}
\hline \multirow{2}{*}{$\begin{array}{l}\text { Parâmetros } \\
\text { genéticos }^{(1)}\end{array}$} & \multicolumn{3}{|c|}{ Peso (g) } & \multirow{2}{*}{$\begin{array}{c}\text { Teor de } \\
\text { óleo }(\%)\end{array}$} \\
\hline & Casca & Amêndoa & Grãos & \\
\hline$\overline{\hat{\sigma}_{g p}^{2}}$ & 0,0580 & 0,3398 & 0,6560 & 0,0002 \\
\hline$\hat{\sigma}^{2}$ & 0,0539 & 0,3000 & 0,5440 & 0,0003 \\
\hline$\hat{\sigma}_{\mathrm{f}}^{2}$ & 0,1119 & 0,6399 & 1,2001 & 0,0005 \\
\hline$\hat{\rho}_{\mathrm{i}}$ & $0,516 \pm 0,094$ & $0,531 \pm 0,085$ & $0,546 \pm 0,096$ & $0,425 \pm 0,084$ \\
\hline$\rho_{\text {im }}$ & 0,6830 & 0,6937 & 0,7069 & 0,5970 \\
\hline$\underline{\mathrm{r}_{\mathrm{pp}}^{\wedge}}$ & 0,8264 & 0,8329 & 0,8408 & 0,7726 \\
\hline Média & 4,3498 & 7,2186 & 11,5686 & 32,62 \\
\hline
\end{tabular}

de ganhos com a seleção. Observou-se maior acréscimo no valor dessa estimativa com a seleção baseada na informação de duas colheitas, e duas medições foram suficientes para obtenção de estimativas de acurácia de seleção próximas a $80 \%$ (Tabela 4 ).

A decomposição das relações de causa e efeito entre as características produtivas e de qualidade de grãos tem mostrado que não existe associação entre esses componentes do rendimento de óleo (Heller, 1996; Rao et al., 2008; Spinelli et al., 2010). Para selecionar matrizes com qualidade superior de grãos, a serem recombinadas com genótipos de maior produção de grãos, considerou-se uma intensidade de seleção de 10\% (Resende, 2002).

A seleção de genótipos superiores pode se basear em uma única característica ou considerar um conjunto de atributos favoráveis para o desenvolvimento de um produto final de qualidade superior. $\mathrm{O}$ ganho da seleção direta em todos os caracteres foi superior ao ganho indireto, tendo apresentado menor diferença quando as características foram correlacionadas, como peso de amêndoa e teor de óleo (Tabela 5). De acordo com Falconer (1981), a seleção indireta pode promover ganhos maiores do que a seleção direta, se o caráter auxiliar apresentar maior herdabilidade que o principal, o que não foi observado. Os maiores ganhos individuais foram estimados para peso de amêndoa e teor de óleo.

A seleção direta para menor peso de casca resultou na redução de outros importantes componentes do rendimento de óleo, como peso de grãos e peso de amêndoa. Assim, em virtude da magnitude e do sentido das correlações genéticas, a seleção baseada em uma única característica pode resultar em alterações em outros componentes de produção da planta.

Tabela 5. Estimativas do progresso genético (\%) com o uso de índices de seleção e com a seleção univariada direta e indireta.

\begin{tabular}{|c|c|c|c|c|c|}
\hline \multirow{2}{*}{$\begin{array}{l}\text { Características } \\
\text { principais e índices }\end{array}$} & \multicolumn{3}{|c|}{ Peso } & \multirow{2}{*}{$\begin{array}{c}\text { Teor de } \\
\text { óleo }\end{array}$} & \multirow{2}{*}{$\begin{array}{c}\text { Ganhos } \\
\text { totais }\end{array}$} \\
\hline & Casca & Amêndoa & Grãos & & \\
\hline & \multicolumn{5}{|c|}{ Ganhos de seleção diretos e indiretos } \\
\hline Peso de casca & $-11,47^{(1)}$ & $-15,42$ & $-14,12$ & 1,83 & $-16,24$ \\
\hline Peso de amêndoa & 6,17 & $11,95(1)$ & 9,91 & 4,94 & 20,63 \\
\hline Peso total & 6,69 & 11,83 & $10,04^{(1)}$ & 4,53 & 19,71 \\
\hline \multirow[t]{2}{*}{ Teor de óleo } & 0,86 & 2,95 & 2,19 & $10,06^{(1)}$ & 14,34 \\
\hline & \multicolumn{5}{|c|}{ Ganhos de seleção com uso de índices de seleção } \\
\hline Genótipo ideótipo & 3,27 & 9,65 & 8,02 & 6,28 & 20,68 \\
\hline Elston (1963) & 5,10 & 10,08 & 8,44 & 3,52 & 16,94 \\
\hline Mulamba \& Mock (1978) & 3,25 & 8,29 & 6,56 & 4,78 & 16,38 \\
\hline
\end{tabular}

${ }^{(1)}$ Valores representam o ganho com a seleção direta. 
Os ganhos totais obtidos pelos índices foram semelhantes aos obtidos pela seleção direta, com uma pequena vantagem (Tabela 5). Notadamente, observou-se que os índices permitiram selecionar plantas de maior teor de óleo e de maior peso de grãos. O índice genótipo-ideótipo foi o que resultou em maior ganho total (20,68\%), tendo promovido alteração mais equilibrada na média das características, uma vez que os ganhos indiretos foram semelhantes aos obtidos pela seleção direta. Os maiores ganhos totais obtidos com esse índice foram verificados para peso de grãos $(8,02 \%)$, peso de amêndoa $(9,65 \%)$ e teor de óleo $(6,28 \%)$.

O ganho total obtido com a seleção direta para peso de amêndoa foi comparável aos ganhos observados com a utilização dos índices de seleção (Tabela 5). Portanto, a associação de maior facilidade de avaliação dessa característica aos ganhos indiretos estimados contribui para que o peso de amêndoa se torne um importante critério de seleção.

Em contraste com a baixa diversidade genética quantificada por técnicas de marcadores moleculares (Rosado et al., 2010), avaliações agronômicas têm mostrado variabilidade genética para os principais componentes de produção dessa oleaginosa (Mishra et al., 2009; Freitas et al., 2011). Ganhos de seleção superiores a $80 \%$ foram estimados por Drumond et al. (2010) e Laviola et al. (2010), na seleção para produtividade de grãos. No entanto, embora os parâmetros genéticos indiquem predominância da variação genotípica, o progresso genético por meio da seleção quanto à qualidade de grãos pode ser considerado limitado. Assim, torna-se necessária a avaliação de novos acessos, bem como a realização de cruzamentos, para geração de variabilidade, e a seleção de plantas que reúnam um conjunto de caracteres favoráveis.

\section{Conclusões}

1. Os parâmetros genéticos, na população avaliada de pinhão-manso, apresentam predominância da variação genotípica na expressão de características de qualidade de grãos, com tendência de manutenção da superioridade dos genótipos ao longo do tempo.

2. Para obtenção de valores de acurácia seletiva próximos a $80 \%$, são necessárias duas medições.

3. O ganho por meio da seleção direta, em todos os caracteres, é superior ou comparável ao ganho indireto.
4. O índice genótipo-ideótipo possibilita maior ganho total na seleção e proporciona alteração mais equilibrada na média das características.

5. A seleção direta quanto ao peso de amêndoas, como caráter principal, resulta em ganhos totais semelhantes aos obtidos com a utilização dos índices de seleção.

\section{Referências}

ACTHEN, W.M.J.; NIELSEN, L.R.; AERTS, R.; LENGKEEK, A.G.; KJAER, E.D.; TRABUCCO, A.; HANSEN, J.K.; MAES, W.H.; GRAUDAL, L.; AKINNIFESI, F.K.; MUYS, B. Towards domestication of Jatropha curcas. Biofuels, v.1, p.91-107, 2010.

ARRUDA, F.P. de; BELTRÃO, N.E. de M.; ANDRADE, A.P. de; PEREIRA, W.E.; SEVERINO, L.S. Cultivo de pinhão-manso (Jatropha curca L.) como alternativa para o semi-árido nordestino. Revista Brasileira de Oleaginosas e Fibrosas, v.8, p.789-799, 2004.

CRUZ C.D.; REGAZZI A.J.; CARNEIRO P.C.S. Modelos biométricos aplicados ao melhoramento genético. Viçosa: UFV, 2004. 480p.

DRUMOND, M.A.; SANTOS, C.A.F.; OLIVEIRA, V.R. de; MARTINS, J.C.; ANJOS, J.B. dos; EVANGELISTA, M.R.V. Desempenho agronômico de genótipos de pinhão manso no Semiárido pernambucano. Ciência Rural, v.40, p.44-47, 2010.

ELSTON, R.C.A. Weight-free index for the purpose of ranking or selection with respect to several traits at a time. Biometrics, v.19, p.85-97, 1963.

FALCONER, D.S. Introdução à genética quantitativa. Viçosa: UFV, 1981. 279p.

FREITAS, R.G.; MISSIO, R.F.; MATOS, F.S.; RESENDE, M.D.V.; DIAS, L.A.S. Genetic evaluation of Jatropha curcas: an important oilseed for biodiesel production. Genetics and Molecular Research, v.10, p.1490-1498, 2011.

GINWAL H.S.; PHARTYAL S.S.; RAWAT P.S.; SRIVASTAVA R.L. Seed source variation in morphology, germination and seedling growth of Jatropha curcas (L.), in Central India. Silvae Genetica, v.54, p.76-80, 2005.

HELLER, J. Physic nut (Jatropha curcas L.): promoting the conservation and use of underutilized and neglected crops. Rome: Institute of Plant Genetics and Crop Plant Research, 1996. 66p.

INSTITUTO ADOLFO LUTZ. Normas analíticas do Instituto Adolfo Lutz. 3.ed. São Paulo: IMESP, 1985. v.1, 533p.

KAUSHIK, N.; KUMAR, K.; KUMAR, S.; KAUSHIK, N.; ROY, $\mathrm{S}$. Genetic variability and divergence studies in seed traits and oil content of Jatropha (Jatropha curcas L.) accessions. Biomass and Bioenergy, v.31, p.497-502, 2007.

LAVIOLA, B.G.; ROSADO, T.B.; BHERING, L.L.; KOBAYASHI, A.K.; RESENDE, M. D.V. Genetic parameters and variability in physic nut accessions during early developmental stages. Pesquisa Agropecuária Brasileira, v.45, p.1117-1123, 2010. 
MISHRA, D.K. Selection of candidate plus phenotypes of Jatropha curcas L. using method of paired comparisons. Biomass and Bioenergy, v.33, p.542-545, 2009.

MULAMBA, N.N.; MOCK, J.J. Improvement of yield potential of the Eto Blanco maize (Zea mays L.) population by breeding for plant traits. Egypt Journal of Genetics and Cytology, v.7, p.40-51, 1978.

NORMAIS climatológicas (1961-1990). Brasília: Secretaria Nacional de Irrigação, Departamento Nacional de Meteorologia, 1992. 84p.

NUNES, C.F.; SANTOS, D.N. dos; PASQUAL, M.; VALENTE, T.C.T. Morfologia externa de frutos, sementes e plântulas de pinhão-manso. Pesquisa Agropecuária Brasileira, v.44, p.207-210, 2009.

OLIVEIRA, A.J. de; RAMALHO, J. (Coord.). Plano Nacional de Agroenergia: 2006-2011. 2.ed. rev. Brasília: Embrapa Informação Tecnológica, 2006. 110p.

QUINTERO, V.P.; ANAYA-LOPEZ, J.L.; COLMENERO, A.Z.; GARCIA, N.M.; COLIN, C.A.N.; BONILLA, J.L.S.; AGUILAR-RANGEL, M.R.; LANGARICA, H.R.G.; BUSTAMANTE, D.J.M. Molecular characterisation of Jatropha curcas L. genetic resources from Chiapas, Mexico through AFLP markers. Biomass and Bioenergy, v.35, p.1897-1905, 2011.

RAO, G.R.; KORWAR, G.R.; SHANKER,A.K.; RAMAKRISHNA, Y.S. Genetic associations, variability and diversity in seed characters, growth, reproductive phenology and yield in Jatropha curcas (L.) accessions. Trees, v.22, p.697-709, 2008.

RESENDE, M.D.V. de. Genética biométrica e estatística no melhoramento de plantas perenes. Brasília: Embrapa Informação Tecnológica; Colombo: Embrapa Florestas, 2002. 975p.

ROCHA, R.B.; MURO-ABAD, J.I.; ARAÚJO, E.F.; CRUZ, C.D. Avaliação do método centróide para estudo de adaptabilidade ao ambiente de clones de Eucalyptus grandis. Ciência Florestal, v.15, p.255-266, 2005.

ROSADO, T.B.; LAVIOLA, B.G.; FARIA, D.A.; PAPPAS, M.R.; BHERING, L.L.; QUIRINO, B.; GRATTAPAGLIA, D. Molecular markers reveal limited genetic diversity in a large germplasm collection of the biofuel crop Jatropha curcas L. in Brazil. Crop Science, v.50, p.2372-2382, 2010.

SPINELLI, V.M.; ROCHA, R.B.; RAMALHO,A.R.; MARCOLAN, A.L.; VIEIRA, J.R.; FERNANDES, C. de F.; MILITÃO, J.S.T.; DIAS, L.A. dos S. Componentes primários e secundários do rendimento de óleo de pinhão-manso (Jatropha curcas L.). Ciência Rural, v.40, p.1752-1758, 2010.

Recebido em 1ํ de outubro de 2011 e aprovado em 29 de dezembro de 2011 